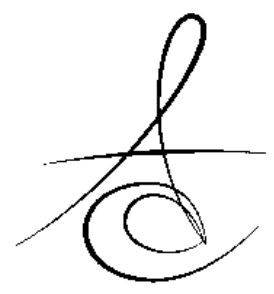

\section{RADİKÜLER DENTİNE ER:YAG LAZER UYGULAMASININ CAD/CAM İLE ÜRETİLMIŞ ZİRKONYUM POSTLARIN OVAL KÖK KANALLARINA PUSH-OUT BAĞLANMA DAYANIMINA ETKİSİ}

\author{
THE EFFECT OF ER:YAG LASER PREATREATMENT OF RADICULAR DENTIN \\ ON THE PUSH-OUT BOND STRENGTH OF CAD/CAM FABRICATED MILLED \\ ZIRCONIA POSTS IN OVAL ROOT CANALS
}

\author{
Dr. Cangül KESKİN*
}

Makale Kodu/Article code: 3694

Makale Gönderilme tarihi: 24.05.2018

Kabul Tarihi: 13.07.2018

\section{öz}

Amaç: $\mathrm{Bu}$ orijinal araştırmanın amacı, kök duvarlarında Er:YAG lazer kullanımının, oval kök kanallarında açılan post boşluklarına özel olarak Bilgisayar Yardımıyla Tasarım / Bilgisayar Yardımıyla Üretim (CAD/CAM) teknolojisiyle hazırlanan zirkonyum postların push-out bağlanma dayanımına etkisini değerlendirmektir.

Gereç ve Yöntemler: Çalışmaya yapılan radyolojik muayene sonucunda seçilen, oval kök kanal konfigürasyonuna sahip 40 diş dahil edildi. Kök kanal tedavisinin tamamlanmasının ardından ultrasonik uçlarla post boşluğu hazırlandı. Örnekler rastgele şekilde, post boşluğu duvarlarına Er:YAG uygulanıp uygulanmamasına göre iki gruba ayrıldı $(n=20)$. Her bir örnek için post boşluğunun taranmasıyla freze cihazıyla eksiltme yöntemi ile yttria stabilize tetragonal zirkonyum polikristal (Y-TZP) bloklarından zirkonya postlar üretildi. Postlar sinterlendikten sonra simante edildi. Push-out bağlanma testi uygulandı ve elde edilen veriler t-testi ile chi-square testi kullanılarak istatistiksel olarak değerlendirildi.

Bulgular: Kök kanal duvarlarına Er:YAG lazer uygulaması yapılmış olan grubun push-out bağlantı değerleri, lazer uygulamayan gruba göre anlamlı oranda yüksek bulunmuştur $(p<0.05)$.

Sonuç: Radiküler dentine Er:YAG lazer uygulaması push-out bağlanma değerlerini arttırmıştır.

Anahtar kelimeler: Endodonti, Diş restorasyonu, Zirkonyum post, Er:YAG lazer

\section{ABSTRACT}

Aim: This original research study aimed to evaluate the effect of Er:YAG laser irradiation of post spaces prepared in oval root canals on the push-out bond strenght of zirconia posts manufactured by Computer Aided Design / Computer Aided Manufacturing (CAD/CAM) technology.

Materials and Methods: Forty teeth showing oval root canal configuration were included to the study following the radiological examination. Post spaces were prepared with ultrasonic tips after the completion of root canal treatment. Specimens were divided into two groups randomly either they were irradiated with Er:YAG lasers or not $(n=20)$. For each specimen, zirconium posts were produced by milling from yttria-tetragonal zirconia polycrystal (Y-TZP) blocks. Posts were sintered and luted in the post spaces of specimens. Push-out bond strenght test was performed and data was statistically analyzed using ttest and chi-square test.

Results: The push-out bond strenght values of the group, which specimens were irradiated with Er:YAG laser were significantly higher when compared to nonlased group $(p<0.05)$.

Conclusion: Er:YAG laser pretreatment of radicular dentin improved the push-out bond strenght values.

Key Words: Endodontics, Dental restoration, Zirconium posts, Er:YAG laser

\footnotetext{
* Ondokuz Mayıs Üniversitesi Diş Hekimliği Fakültesi Endodonti Anabilim Dalı, Samsun
} 


\section{GİRİş}

Kök kanal tedavisi sonrasında uygulanacak olan restorasyonun kalitesi dişin prognozunu önemli ölçüde etkilemektedir. ${ }^{1}$ Kök kanal tedavili bir dişin restorasyonu retansiyon göstermeli, dengeli kuvvet dağıııı sağlamalı ve diş formunu yeniden kazandırmalıdır. $^{2}$ Endodontik tedavi sonrası kronda çürük, eski restorasyon ve travma gibi sebeplerle aşırı madde kaybının görüldüğü durumlarda postlar sıklıkla kullanılmaktadır. ${ }^{3}$ Günümüzde metal postların yerini daha estetik sonuçlar sağlamaları ve daha pratik olmaları nedeniyle fiberle güçlendirilmiş kompozit ve zirkonyum materyallerinden yapılmış postlar almıştır. ${ }^{4}$ Endodonti pratiğinde post restorasyonların en büyük başarısızlık nedenlerini retansiyon kaybı ve kök kırığı oluşturmaktadır. . $^{5,6}$

Post retansiyonu, postun uzunluğuyla, yapıştırma simanının türüyle, post etrafındaki siman kalınlığıyla ve postun türüyle ilişkili bulunmuştur. ${ }^{7-10}$ Çeşitli lazer sistemlerinin post boşluğunu meydana getiren dentin duvarlarına uygulanmasının postların bağlanma dayanımı üzerine etkisi de değerlendirilmiş ve Er:YAG lazer uygulamasının bağlanma dayanımını arttırdığı rapor edilmiştir. ${ }^{11}$

Oval kök kanal konfigürasyonu premolar dişlerde ve mandibular molarların distal kök kanal konfigürasyonlarında sıklıkla görülmektedir. ${ }^{12}$ Oval kök kanal konfigürasyonu, kök kanal sisteminin etkili temizlenmesi ve şekillendirilmesinde yarattığı anatomik zorluklar sebebiyle endodontik tedavi başarısızlığının nedenlerinden biri olarak gösterilmiştir. ${ }^{13}$ Benzer problemlere oval kök kanallarının post ile restorasyonlarında da rastlanmaktadır. Post boşluğu hazırlamak için kullanılan driller yuvarlak kesite sahiptir ve yuvarlak kesitli bir kavite preparasyonu yaparlar. Drillerin oval kanallarda temas etmedikleri bölgelerde rezidüel dolgu materyalleri, debris ve smear tabakası birikmektedir. ${ }^{14}$ Ayrıca drill kullanımı, sağlam dentin dokusunun kaybına yol açarak dişin fraktür dayanımını azaltmaktadır. ${ }^{2}$

Post boşluğu preparasyonu mümkün olduğunca konservatif olmalıdır. Döküm postlar, sağlam dentin dokusunu koruma, adaptasyon ve tutuculuk açısından yuvarlak kesitli prefabrik postlara göre üstün özellikler gösterir. ${ }^{15}$ Ancak geleneksel döküm postların, uzun laboratuvar işlemleri nedeniyle zaman alıcı olmaları, elastisiteleri dentinden yüksek olduğundan ötürü kırıklara yol açmaları ve korozyona uğrayarak estetik beklentileri karşılayamamaları, araştırıcıları farklı post sistemlerine yönlendirmiştir. ${ }^{16-18}$ Seramikler, estetik, biyouyumlu ve yüksek kırılma dayanımına sahip materyaller olarak post uygulamalarında da kullanılmıştır. ${ }^{19}$ Seramik postlar cam seramik ve zirkonyum esaslı olarak ikiye ayrılırlar. ${ }^{20}$ Zirkonyum oksit esaslı seramikler yüksek kırılma dayanımı göstermekte ve translusensliği sayesinde dentin rengini yansitabilerek estetik restorasyonlara imkan vermektedir. ${ }^{19}$ Zirkonyum oksit esaslı hazır seramik blokların bilgisayar destekli ünitelerle aşındırıldığı CAD/CAM tekniklerinin yaygınlaşmasıyla, zirkonyum oksit esaslı seramiklerin endodontik tedavi sonrası restorasyonlarda kullanımı popüler hale gelmiştir.

Çalışmamızın amacı, post boşluğunun dentin duvarlarına Er:YAG lazer uygulanmasının CAD/CAM teknolojisiyle üretilmiş özel zirkonyum postların pushout bağlanma dayanımına etkisini değerlendirmektir. Çalışmanın boş sıfır hipotezi, lazer uygulanmış örneklerin push-out bağlanma dayanımı değerlerinin, lazer uygulanmamış örneklerden farklı olmadığı şeklinde kurulmuştur.

\section{GEREÇ VE YÖNTEM}

Çalışmada protetik ve/veya periodontal sebeplerle çekilmiş mandibular premolar dişler kullanıldı. Toplanan dişlerin mesiodistal ve bukkolingual doğrultuda radyografileri incelendi ve Wu ve ark.'nın sınıflaması doğrultusunda oval kök kanal konfigürasyonuna sahip 40 adet mandibular premolar diş çalışmaya dahil edildi. ${ }^{13}$ Kök yüzeylerindeki yumuşak ve sert eklentiler kürete edildikten sonra dişler \%1'lik kloramin solüsyonunda dezenfekte edildi. Dişlerin kronları su soğutması altında elmas separey frezleri ile uzaklaştırıldı. Dijital kumpas yardımıyla köklerin uzunlukları ölçülerek ortalama $13 \mathrm{~mm}$ olacak şekilde standardize edildi. Örnekler $4^{\circ} \mathrm{C}^{\prime} \mathrm{de} \% 100$ nemli ortamda muhafaza edildi.

Örneklerin kök kanal tedavileri ve post restorasyonu uygulamaları aynı araştırmacı tarafından gerçekleştirildi. Çalışma boyları 10 nolu K tipi eğe ile apikal foramenden $1 \mathrm{~mm}$ kısa olacak şekilde belirlendi. Kök kanallarının preparasyonu ProTaper Next eğeleri (Dentsply, Maillefer, Ballaigues, İsviçre) ile X3 no'lu eğeye kadar her değişiminde \%5'lik $\mathrm{NaOCl}$ (Sultan, İstanbul, Türkiye) irrigasyonu yapılarak tamamlandı. Preparasyonun ardından kök kanalları sırasıyla \%17'lik 
EDTA (Imıcryl, Konya, Türkiye), distile su ve \%5'lik $\mathrm{NaOCl}$ ile irrige edildi ve steril kağıt konlarla (ProTaper Next paper points, Dentsply, Maillefer) kurulandı. Kök kanal dolguları AH Plus patı (Dentsply, Maillefer) ve güta perka (X3 ana kon ve aksesuar konlar, Dentsply, Maillefer) kullanılarak soğuk lateral kompaksiyon yöntemi ile yapıldı. Kök kanal obturasyonunun kalitesi radyografilerle kontrol edildi. Örnekler patın sertleşmesini sağlamak amacıyla 48 saat $4^{\circ} \mathrm{C}^{\prime}$ de $\% 100$ nemli ortamda muhafaza edildi.

Örneklerin post boşluğu preparasyonunda oval ultrasonik uç (Ellipson, RTD/Satelec, Merignac, Fransa) ultrasonik cihazın (Suprasson, Satelec/Acteon, Group Mount, NJ, ABD) orta güç ayarında su soğutması altında kullanıldı. Üretici firma talimatları doğrultusunda her uç 4 örnekte kullanıldı. Apikalde 5 $\mathrm{mm}$ kök kanal dolgusu kalacak şekilde $8 \mathrm{~mm}$ 'lik post boşlukları hazırlandı. Post boşlukları, debris ve rezidüel kanal dolgusunu uzaklaştırmak için \%17'lik EDTA solüsyonu ve distile su ile irrige edildi ve steril kağıt konlarla kurulandı.

Örnekler ayrı ayrı silikona (Endurent; Zetaplus, Zhermack, İtalya) gömülerek elektronik tarama boyunca sabitlendi. CAM-Stone N (Siladent, Goslar, Almanya) örneklere uygulanarak tarama ve dijital ölçü alma işlemleri tamamland. Cerec InLab 3D 3.03 programı (Sirona Dental Systems, GmbH, Bensheim, Almanya) ile örneklerin ve post restorasyonlarının 3 boyutlu rekonstrüksiyonu yapıldı. Her örneğe özel zirkonyum postlar Y-TZP bloklardan (inCoris ZI, Sirona Dental Systems, GmbH, Bensheim, Almanya) eksiltme tekniği ile prepare edildi ve $1500^{\circ} \mathrm{C}^{\prime}$ de 8 saat boyunca sinterlendi (Resim1,2,3).

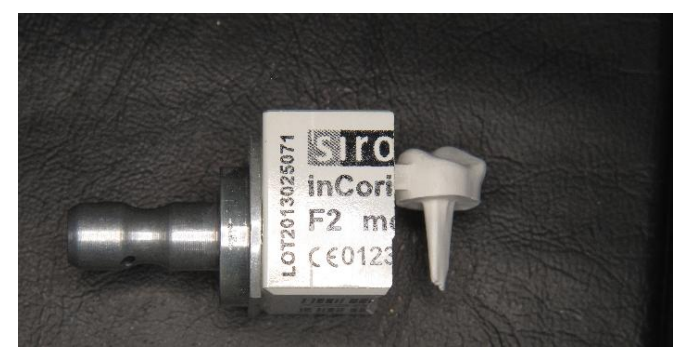

Resim 1. Zirkon bloğun Cad/Cam cihazında üretim sonrası görüntüsü.

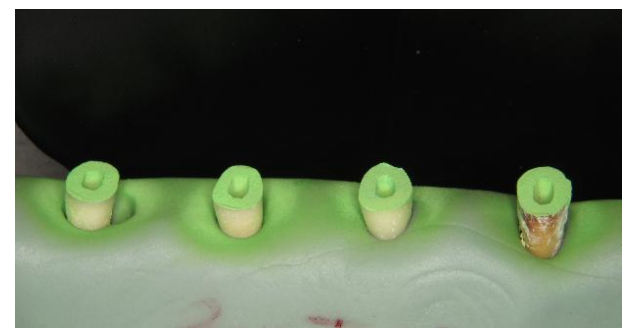

Resim 2. Tarama ve dijital ölçü almak için silikona sabitlenmiş örneklerin görüntüsü

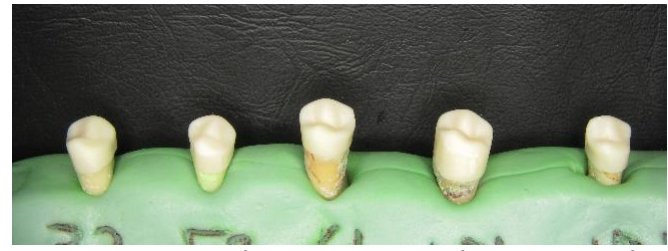

Resim 3. Sinterleme sonrası Zirkonyum postların uyum modeli.

Örnekler Er:YAG lazer uygulanmasına göre rastgele 2 gruba ayrıldı $(n=20)$. Grup 1'deki örneklerin post boşluklarına $2.940 \mathrm{~nm}$ dalga boyu ve $15 \mathrm{~W}$ güç parametreleri kullanılarak Er:YAG lazer (Doctor Smile; Lambda Scientifica Srl, Vicenza, İtalya) uygulandı. $400 \mu \mathrm{m}^{\prime}$ lik uç post boşluğuna yerleştirildi ve lazer sistemi toplam 60 saniye aktif, $100 \mu$ saniye pasif şekilde aralıklı modda çalıştırıldı. Grup 2'deki örneklere herhangi bir işlem yapılmadı ve örnekler push-out testi uygulanana kadar $4^{\circ} \mathrm{C}$ 'de $\% 100$ nemli ortamda muhafaza edildi.

Self-adesiv rezin siman (RelyX Unicem, 3M ESPE, Seefeld, Almanya) üretici firmanın talimatlarına göre siman sisteminin özel endodontik uçları kullanılarak post boşluklarına uygulandı. Postlar hafif parmak baskısıyla post boşluklarına yerleştirildi ve simanın fotoaktivasyonu, LED ışık cihazı (Elipar S10, 3M ESPE, Seefeld, Almanya) standart modda (750 $\mathrm{mW} / \mathrm{cm}^{2}$ yoğunlukta) kullanılarak örneğin koronal yüzeyinden toplam 40 saniyede tamamlandı. Örnekler 24 saat $37^{\circ} \mathrm{C}^{\prime}$ de distile su içinde muhafaza edildi.

Push-out testi için her bir örnekten düşük hızlı elmas testere (Isomet, Buehler, IL, ABD) ile su soğutması altında $1 \mathrm{~mm}$ kalınlığında 4-6 adet kesitler alındı. Alınan her kesitin koronal yüzeyi kalemle işaretlendi. Testten önce postların yüzey alanının hesaplanması için kesitlerin görüntüleri alındı ve Coniglio ve ark.'nın oval postlar için önerdiği formüle göre kesit yüzey alanları hesaplandı. ${ }^{21}$ Push-out

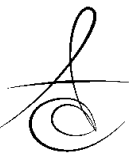


bağlanma dayanımı testi için Universal test cihazı (Instron Universal test cihazı, Elista, İstanbul, Türkiye) kullanıldı. Kuvvet, yönü apikalden koronale olacak şekilde, $0.5 \mathrm{~mm} / \mathrm{dk}$ hızında her bir post kök kanalından ayrılana kadar uygulandı. Örnekler kırıma tiplerinin değerlendirilmesi için tekrar görüntülendi ve kırılma tipleri adesiv, kohesiv ve karışık olarak sınıflandırıldı. Elde edilen verilerin istatistiksel analizi SPSS programında (Chicago, IL, ABD) t-testi ve ki-kare testi kullanılarak yapıldı.

\section{BULGULAR}

Grup 1 Lazer uygulanan ve Lazer uygulanmayan Grup 2 gruplar arasında postların pushout bağlanma dayanımları arasında istatistiksel olarak anlamlı farklılık saptanmıştır $(p<0.05)$ (Tablo 1). Post boşluklarına Er:YAG lazer uygulanmış olan Grup 1 lazer uygulanan grubun push-out bağlanma dayanımı değerleri lazer uygulanmayan gruptan anlamlı oranda yüksek bulunmuştur.

Tablo 1. Deney gruplarının Push-out bağlanma dayanımlarının ortalama ve standart deviasyon değerleri (MPa)

\begin{tabular}{llc}
\hline Deney grupları & $\begin{array}{l}\text { Push-out Bağlanma Dayanımı } \\
\text { Ortalama } \pm \text { SD (MPa) }\end{array}$ & $\mathrm{N}$ \\
\hline $\begin{array}{l}\text { Lazer Uygulanan Grup } \\
\text { Grup 1 }\end{array}$ & $12.06 \pm 1.252$ & 20 \\
\hline $\begin{array}{l}\text { Lazer Uygulanmayan } \\
\text { Grup } \\
\text { Grup 2 }\end{array}$ & $9.947 \pm 1.916$ & 20 \\
\hline
\end{tabular}

Gruplar koronal ve apikal bölge olarak alt gruplara ayrıldığında lazer uygulanan postların pushout bağlanma dayanımları arasında her iki bölgede lazer uygulanmayan gruba göre istatistiksel olarak anlamlı/önemli bir farklılık oluşmuştur $(p<0.05)$. Lazer uygulanan grubun kendi içinde koronal ve apikal bölgenin push-out bağlanma dayanımları arasında istatistiksel olarak anlamlı/önemli bir farklılık oluşmuştur $(p<0.05)$. Koronal bölgenin push-out bağlanma dayanımları apikal bölgeye göre daha yüksektir. Lazer uygulanmayan grupta koronal ve apikal bölgeler arasında push-out bağlanma dayanımları değerlerinde istatistiksel olarak anlamlı farklılık oluşmamıştır( $p>0.05$ ) (Tablo 2).

Bağlanma dayanımı testinin ardından yapılan incelemede her iki grupta da adesiv tipte başarısızlık en sık görülmüştür. Ancak her iki grupta da adesiv, kohesiv ve karışık tip başarısızlıkların tamamına rastlanmıştır. Ki-kare testi sonucunda gruplar arasında başarısızlık şekli açısından anlamlı fark saptanmamıştır $(p>0.05)$.

Tablo 2. Deney gruplarının koronal ve apikal push-out bağlanma dayanımlarının ortalama ve standart deviasyon değerleri $(\mathrm{MPa})$

\begin{tabular}{llllll}
\hline Bölgeler & \multicolumn{3}{l}{ Koronal Bölge } & Apikal Bölge \\
\hline Push-out Bağlanma Dayanımı & $N$ & Mean & SD (MPa) & Mean & SD (MPa) \\
\hline Lazer Uygulanan Grup & 20 & 0.080 & 0.154 & 0.059 & 0.013 \\
\hline Lazer Uygulanmayan Grup & 20 & 0.046 & 0.194 & 0.043 & 0.143 \\
\hline
\end{tabular}

\section{TARTIŞMA}

Günümüze kadar post restorasyonlarının üretiminde çeşitli materyaller kullanılmıştır. Metal postların estetik olarak kritik bölgelerde meydana getirdikleri sorunlar metal olmayan fiber, seramik ve zirkonya postların geliştirilmesini sağlamıştır. ${ }^{4}$ Zirkonya üstün estetik ve mekanik özellikleri bakımından tercih edilmektedir. ${ }^{19,22}$ CAD/CAM freze tekniği ile üretilen zirkonya post sistemleri de güncel olarak uygulanmaktadır. ${ }^{19,22} \mathrm{Bu}$ çalışmanın sonuçlarına göre post boşluğundaki radiküler dentine Er:YAG lazer uygulanması zirkonyum postların push-out bağlanma dayanımını anlamlı oranda etkilemektedir. Dolayısıyla boş hipotez ret edilmiştir.

Post restorasyonu ile radiküler dentin arasındaki bağlantı dayanımı pull-out, mikrogerilim ve push-out testleri ile ölçülebilmektedir. 6,9,23 Mikrogerilim bağlanma dayanımı testlerinin en büyük dezavantajı yüksek prematür başarısızlık oranıdır. ${ }^{24}$ Pull-out bağlanma dayanımı testleri kök yüzeyinin tamamı boyunca olan bağlanma dayanımını ölçerek farklı bölgelerin kıyaslanmasına imkan tanımaz. Push-out testleri güvenilir ve doğru sonuçlar vermelerinden ötürü post retansiyonunu değerlendirmek için en uygun yöntem olarak rapor edilmiştir. ${ }^{24}$ Push-out bağlanma testlerinde kesitler üzerinde klinik koşulları simüle edecek şekilde kuvvet uygulanabilir. ${ }^{25}$ Kesit kalınlığının testin duyarlılığını etkilediği; kalın kesitlerin hatalı sonuçlara yol açabilecek uniform olmayan stres dağılımlarına sebep oldukları belirtilmiştir. ${ }^{26} \mathrm{Bu}$ sınırlamayı ortadan kaldırmak için kesitlerin $1 \mathrm{~mm}$ kalınlığında prepare edilmesi gerektiği bildirilmiştir. ${ }^{27}$

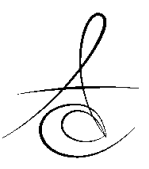


Çalışmamızda bu amaçla ince kesit push-out bağlanma dayanımı testi uygulanmıştır.

Restoratif materyallerin dentine bağlantısı operatörün tecrübesinden etkilenen hassas bir prosedürdür. Hekim tecrübesi arttıkça bağlanma dayanımı değerlerinin de arttı̆ı̆ bildirilmiştir. ${ }^{28}$ Bu faktörün standardize edilebilmesi için, çalışmamızda örneklerin preparasyonu, restorasyonu ve push-out bağlanma testi uygulamaları, dental adesivlerin ve kanal içi postların uygulanmasında klinik tecrübesi olan endodonti uzmanı olan iki araştıııı tarafından yapılmıştır.

Er:YAG lazerler, dental sert dokuların su içeriği üzerine termomekanik etki göstererek organik dokuların ablasyonuna sebep olurlar. ${ }^{29}$ Uygulama sırasında meydana gelen mikro patlamalar dentin tübüllerindeki inorganik ve organik dokunun açı̆̆a çıkarılmasına dolayısıyla dentin tübüllerinin ve tübül ağızlarının temizlenmesine yol açar. ${ }^{29,30} \mathrm{Bu}$ çalışmada, post boşluğu dentin duvarlarına Er:YAG lazer uygulanması zirkonyum postlar ile radiküler dentin arasındaki pushout bağlanma dayanımını anlamlı oranda arttırmıştır. Bu artış, Er:YAG uygulaması sonucu dentinde meydana gelen mikro yapısal değişikliklere bağlanabilir. Er:YAG lazerlerin dentin üzerindeki yapısal etkilerine ek olarak, smear tabakasını uzaklaştırdıkları rapor edilmiştir. ${ }^{30}$ Dolayısıyla Er:YAG lazerlerin dentinin permeabilitesini arttırdığı bu nedenle post-dentin bağlanma dayanımının arttığı öne sürülebilir. RelyX Unicem diş dokularına hem kimyasal olarak hidroksiapatitteki kalsiyum iyonlarıla reaksiyona girerek, hem de mekanik olarak rezin taglar ve lateral kökler meydana getirerek bağlanır. ${ }^{31} \mathrm{Bu}$ rezin tagların sayısında ve/veya derinliğindeki artış rezin siman ile dentin yapısı arasındaki mikromekanik bağlanma dayanımı arttırabilir. Post boşluğu preparasyonunun oval ultrasonik uçlarla yapılmasının smear tabakasını uzaklaştırma potansiyeli araştırımışır. Scotti ve ark., ultrasonik uçların da geleneksel drillerin de farklı kalınlıklarda smear tabakası meydana gelmesine yol açtığını rapor etmiştir. ${ }^{32}$ Ultrasonik uçların dentin duvarı yüzeyinde sürtünme etkisiyle debrisi tübüllere ittiği öne sürülmüştür. Ancak Coniglio ve ark. tarafından yapılmış bir taramalı elektron mikroskop çalışması oval ultrasonik uçların smear tabakasını etkili bir şekilde uzaklaştırdığını rapor etmişlerdir. ${ }^{14}$ Çalışmamızda post boşluğu hazırlamada kullanılan ultrasonik uçlar Er:YAG lazer uygulamasının smear tabakası uzaklaştırıması etkinliğine katkı sağlamış olabilir.
Lazer uygulanan örneklerde Push-out bağlanma testlerinde koronal bölge apical bölgeye göre daha yüksek değerler vermiştir. Bunun sebebi olarak yapışırma ajanlarının koronal bölgede daha ince apikale gittikçe daha kalın olmasılya açıklanabilir. ${ }^{33,34}$ Ayrıca Cad/Cam sisteminin sınırlı derinlik taraması, ${ }^{35}$ dentin tübüllerinin yoğunluğu ve pozisyonları ${ }^{36}$, led ışık kaynağının uzaklığı apikal bölgede düşük bağlantı değerleri vermesine neden olabilir. ${ }^{37}$

Lazer uygulamasının etkinliği uygulama süresi ve güç parametrelerine bağlıdır. ${ }^{33,38}$ Er:YAG lazer uygulamasının süresinin 20 saniye olarak belirlendiği bir çalışmada, lazer uygulamasının smear tabakası uzaklaştırmada etkisiz olduğu rapor da edilmiştir. ${ }^{3439}$ Çalışmamızda lazer uygulama süresi 60 saniye olarak belirlenmiş̧ir.

Yapışııma ajanlarının zirkonyum postlara retansiyonu diğer post tipleriyle karşılaştırıldığında göreceli olarak düşük bulunmuştur. ${ }^{3540}$ Zirkonyum postların adesiv rezin yapıştırma simanları ile bağlantısının cam iyonomer veya rezin modifiye cam iyonomer yapıştırma ajanları ile olan bağlantısından güçlü olduğu rapor edilmiştir. ${ }^{36,37,41,42}$ Çalışmamızda kullanılan RelyX Unicem, silika, cam, kalsiyum hidroksit, metrakrilat fosforik ester, dimetakrilat ve asetat içeren self-adesiv rezin yapıda olduğu için tercih edilmiştir. ${ }^{31}$

Çalışmamızın sonuçlarına göre post boşluğu dentin duvarlarına Er:YAG lazer uygulanması CAD/CAM teknolojisi ile vakaya özel olarak üretilmiş zirkonyum postların radiküler dentine push-out bağlanma dayanımını arttırmak için uygulanabilir.

İsmail Uzun: ORCID ID: 0000-0003-3353-3260

Cangül Keskin: ORCID ID: 0000-0001-8990-4847

\section{KAYNAKLAR}

1. Hommez GM, Coppens CR, De Moor RJ. Periapical health related to the quality of coronal restorations and root fillings. Int Endod J 2002; 35: 680-9.

2. Dietschi D, Duc O, Krejci I, Sadan A. Biomechanical considerations for the restoration of endodontically treated teeth: a systematic review of the literature, Part II (Evaluation of fatigue behavior, interfaces, and in vivo studies). Quintessence Int 2008; 39: 117-29.

3. Schwartz RS, Robbins JW. Post placement and restoration of endodontically treated teeth: a literature review. J Endod 2004; 30: 289-301. 
4. Ferrari M. Fiber Posts and endodontically treated teeth: a compendium of scientific and clinical perspectives. Wendywood, South Africa: Modern Dentistry Media 2008.

5. Hatzikyriakos AH, Reisis GI, Tsingos N. A 3-year postoperative clinical evaluation of posts and cores beneath existing crowns. J Prosthet Dent 1992; 67: 454-8.

6. Kremeier K, Fasen L, Klaiber B, Hofmann N. Influence of endodontic post type (glass fiber, quartz fiber or gold) and luting material on pushout bond strength to dentin in vitro. Dent Mater 2008; 24: 660-6.

7. Standlee JP, Caputo AA, Hanson EC. Retention of endodontic dowels: effects of cement, dowel length, diameter, and design. J Prosthet Dent 1978; 39: 401-5.

8. Zicari F, Couthino E, De Munck J, Poitevin A, Scotti R, Naert I, Van Meerbeek B. Bonding effectiveness and sealing ability of fiber-post bonding. Dent Mater 2008; 24: 967-77.

9. D'Arcangelo C, Cinelli M, De Angelis F, D'Amario. The effect of resin cement film thickness on the pull out strength of a fiber-reinforced post system. J Prosthet Dent 2007; 98: 193-8.

10. Perez BE, Barbosa SE, Melo RM, Zamboni SC, Ozcan M, Valandro LF, Bottino MA. Does the thickness of the resin cement affect the bond strength of a fiber post to the root dentin? Int J Prosthodont 2005; 19: 606-9.

11. Nagase DY, de Freitas PM, Morimoto S, Oda M, Vieira GF. Influence of laser irradiation on fiber post retention. Lasers Med Sci 2011; 26: 377-80.

12. Wu MK, R'oris A, Barkis D, Wesselink PR. Prevalence and extent of long oval canals in the apical third. Oral Surg Oral Med Oral Pathol Oral Radiol Endod 2000; 89: 739-43.

13. Wu MK, Wesselink PR. A primary observation on the preparation and obturation of oval canals. Int Endod J 2001; 34: 137-41.

14. Coniglio I, Carvalho CA, Magni E, Cantoro A, Ferrari M. Post space debridement in oval-shaped canals: the use of a new ultrasonic tip with oval section. J Endod 2008; 34: 752-5.

15. Zuckerman GR. Practical considerations and technical procedures for post-retained restorations. J Prosthet Dent 1996; 75: 135-9.
16. Korn SB, von Fraunhofer JA, Mueninghoff LA. An in vitro comparasion of two dowel and core techniques for endodontically treated molars. J Prosthet Dent 1984; 51: 509-14.

17. Eskitaşçıoğlu G, Belli $S$, Kalkan M. Evaluation of two post core systems using two different methods. J Endod 2002; 28: 629-33

18. Butz F, Lenon AM, Heydecke G. Survival rate and fracture strength of endodontically treated teeth maxillary incisors with moderate defects restored with different post-and-core systems: an in vitro study. Int J Prosthodont 2001; 14: 58-64.

19. Özkurt Z, İşeri U, Kazazoğlu E. Zirconia ceramic post systems: a literature review and a case report. Dent Mater J 2010; 29: 233-45.

20. Koutayas SO, Kern M. All ceramic posts and cores: the state of the art. Quintessence Int 1999; 30: 383-92.

21. Coniglio I, Magni E, Cantoro A, Goracci C, Ferrari M. Push-out bond strength of circular and ovalshaped fiber posts. Clin Oral Invest 2011; 15: 66772.

22. Durkan R, Deste G, Şimşek H. Monolitik zirkonya seramik sistemlerinin üretim tipleri ile aşınma, optik ve estetik özellikleri. Atatürk Üniv. Diş Hek. Fak. Derg. 2018; 28: 263-70.

23. Ari $\mathrm{H}$, Yasar A, Belli S. Effects of $\mathrm{NaOCl}$ on bond strengths of resin cements to root canal dentin. J Endod 2009; 29: 248-51.

24. Goracci C, Tavares AU, Fabianelli A, Monticelli F, Raffaelli O, Cardoso PC, Tay F, Ferrari M. The adhesion between fiber posts and root canal walls: comparison between microtensile and push-out bond strength measurements. Eur J Oral Sci 2004; 112: 353-61.

25. Sunsangiam S, van Noort R. Do dentin bond strength tests serve a useful purpose? J Adhes Dent 1999; 1: 57-67.

26. Ngoh EC, Pashley DH, Loushine RJ, Weller N, Kimbrough F. Effects of eugenol on resin bond strengths to root canal dentin. J Endod 2001; 27: 411-4.

27. Coniglio I, Magni E, Cantoro A, Goracci C, Ferrari M. Push-out bond strength of circular and ovalshaped fiber posts. Clin Oral Invest 2011; 15: 66772.

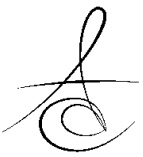


28. Unlu N, Gunal S, Ulker M, Ozer F, Blatz MB. Influence of operator experience on in vitro bond strength of dentin adhesives. J Adhes Dent 2012; 14: 223-7.

29. Tachibana A, Marques MM, Soler JM, Matos AB. Erbium, chromium: yttrium scandium gallium garnet laser for caries removal: influence on bonding of a self-etching adhesive system. Lasers Med Sci 2008; 23: 435-41.

30. Minas $\mathrm{NH}$, Gutknecht $\mathrm{N}$, Lampert F. In vitro investigation of intra-canal dentine-laser beam interaction aspects: II. Evaluation of ablation zone extent and morphology. Lasers Med Sci 2010; 25: 867-72.

31. Gerth HU, Dammaschke T, Züchner H, Schäfer E. Chemical analysis and bonding reaction of RelyX Unicem and Bifix composites-a comparative study. Dent Mater 2006; 22: 934-41.

32. Scotti N,Foniglia A, Bergantin D, Paolino DS, Pasqualini, Berutti E. Fibre post adaptation and bond strength in oval canals. Int Endod J 2014; 47: $366-72$.

33. D'Arcangelo $C$, Cinelli $M$, De Angelis $F$, D'Amario $M$. The effect of resin cement film thickness on the pull out strength of a fiber-reinforced post system. J Prosthet Dent 2007; 98: 193-8.

34. Scotti N, Forniglia A, Bergantin E, Paolino DS, Pasqualini D, Berutti E. Fibre post adaptation and bond strength in oval canals. Int Endod J 2014; 47: 366-72.

35. Shemesh H, Wu MK, Wesselink PR. Leakage along apical root fillings with and without smear layer using two different leakage models: a two-month longitudinal ex vivo study. Int Endod J 2006; 39: 968-76.

36. Tay FR, Loushine RJ, Lambrechts $P$, Weller RN, Pashley $\mathrm{DH}$. Geometric factors affecting dentin bonding in root canals: a theoretical modeling approach. J Endod 2005; 31: 584-9.

37. Goracci C, Corciolani G, Vichi A, Ferrari M. Lighttransmitting ability of marketed fiber posts. $J$ Dent Reserch 2008; 87,1122-6.

38. C. Goya, R. Yamazaki, Y. Tomita, Y. Kimura, K. Matsumoto, "Effects of pulsed Nd: YAG laser irradiation on smear layer at the apical stop and apical leakage after obturation," Int Endod J 2000; 33: 266-71.
39. B. H. Kivanc, Ö. İ. A. Ulusoy, G. Görgül. Effects of Er: YAG laser and Nd: YAG laser treatment on the root canal dentin of human teeth: a SEM study. Lasers Med Sci 2008; 23: 247-52.

40. Rosentritt M, Fürer C, Behr M, Lang R, Handel G. Comparison of in vitro fracture strength of metallic and tooth-coloured posts and cores. J Oral Rehabil 2000; 27: 595-601.

41. Osorio R, Oyagüe RC, Monticelli F, Osorio E, Toledano $M$. Resistance to bond degradation between dual-cure resin cements and pre-treated sintered CAD-CAM dental ceramics. Med Oral Patol Oral Cir Bucal 2012; 17: 669.

42. Oyagüe RC, Monticelli F, Toledano M, Osorio $E$, Ferrari M, Osorio R. Effect of water aging on microtensile bond strength of dual-cured resin cements to pre-treated sintered zirconium-oxide ceramics. Dent Mater 2009; 25: 392-9.

\author{
Yazışma Adresi \\ Doç. Dr. İsmail UZUN \\ Ondokuz Mayıs Üniversitesi \\ Diş Hekimliği Fakültesi \\ Endodonti Anabilim Dalı \\ SAMSUN \\ Tel: 05332342355 \\ E-mail: ismail.uzun@omu.edu.tr
}

